PROCEEDINGS OF THE

AMERICAN MATHEMATICAL SOCIETY

Volume 133, Number 7, Pages 2183-2192

S 0002-9939(05)07802-0

Article electronically published on February 15, 2005

\title{
BOUNDEDNESS OF THE FIRST EIGENVALUE OF THE $p$-LAPLACIAN
}

\author{
ANA-MARIA MATEI
}

(Communicated by Jozef Dodziuk)

\begin{abstract}
We prove that for any $p>1$, any compact manifold of three or more dimensions carries Riemannian metrics of volume one with the first eigenvalue of the $p$-Laplacian arbitrarily large.
\end{abstract}

\section{INTRODUCTION}

The $p$-Laplace operator has been extensively studied in recent years, especially in the context of a bounded domain in $\mathbb{R}^{n}$. Recently, there has been an increasing interest in the study of this operator - and in particular of its first eigenvalue - in the more general setting of Riemannian manifolds.

Let $M$ be a compact connected manifold. The $p$-Laplace operator $(p>1)$ associated to a Riemannian metric $g$ on $M$ is given by

$$
\Delta_{p} f:=\delta\left(|d f|^{p-2} d f\right),
$$

where $\delta=-d i v_{g}$ is the adjoint of $d$ for the $L^{2}$-norm induced by $g$ on the space of differential forms. This operator can be viewed as a natural generalization of the well-known Laplace-Beltrami operator which corresponds to $p=2$. When $\partial M=\emptyset$, the nonlinear partial differential equation

$$
\Delta_{p} f=\lambda|f|^{p-2} f
$$

characterizes the critical points of the $p$-energy functional $E_{p}(f)=\int_{M}|d f|^{p} \nu_{g}$ under the constraint $\int_{M}|f|^{p} \nu_{g}=1\left(\nu_{g}\right.$ denotes the Riemannian volume element induced by $g$ ).

The real numbers $\lambda$ for which this equation has nontrivial solutions are called eigenvalues of $\Delta_{p}$. They represent the critical energy levels. The associated solutions, i.e. the critical functions, are called eigenfunctions.

Obviously, zero is an eigenvalue of $\Delta_{p}$, the associated eigenfunctions being the constant functions. The set $\sigma_{p}(M, g)$ of the nonzero eigenvalues is a nonempty, unbounded subset of $(0, \infty)$ [6]. Its infimum $\lambda_{1, p}(M, g)=\inf \sigma_{p}(M, g)$ is itself a positive eigenvalue called the first eigenvalue of $\Delta_{p}$ and has the following variational characterization [14] :

$$
\lambda_{1, p}(M, g)=\inf \left\{\left.\frac{\int_{M}|d f|^{p} \nu_{g}}{\int_{M}|f|^{p} \nu_{g}}\left|f \in W^{1, p}(M) \backslash\{0\}, \int_{M}\right| f\right|^{p-2} f \nu_{g}=0\right\} .
$$

Received by the editors March 21, 2004 and, in revised form, April 8, 2004.

2000 Mathematics Subject Classification. Primary 58C40; Secondary 58J50.

Key words and phrases. $p$-Laplacian, eigenvalue.

(C) 2005 American Mathematical Society $2183^{\mathrm{R}}$ Reverts to public domain 28 years from publication 
In the present work we consider $\lambda_{1, p}$ as a functional on the space of Riemannian metrics on $M$. Since $\lambda_{1, p}$ is noninvariant under dilatations, we will study the restriction of $\lambda_{1, p}$ to $\mathcal{M}(M)$, the space of Riemannian metrics of volume one on $M$. The question we ask is whether $\lambda_{1, p}$ is bounded on $\mathcal{M}(M)$ ?

A classical elementary argument 3 shows that the infimum of $\lambda_{1,2}$ on this space is zero. The same argument shows that in fact for any $p>1$ the infimum of $\lambda_{1, p}$ on $\mathcal{M}(M)$ is equally zero.

A series of authors (Bérard Bergery and Bourguignon [2, Muto [10, Tanno [11, Urakawa [13]) have showed in various degrees of generality that if $M$ is a sphere of dimension $m \geq 3$, then there is no upper bound for $\lambda_{1,2}$ on $\mathcal{M}\left(S^{m}\right)$. Colbois and Dodziuk [4] (and independently Xu [15]) have extended this result to any compact manifold of dimension $m \geq 3$.

In this paper we prove that this phenomenon is still true for the functional $\lambda_{1, p}$, for any $p>1$.

\section{Preliminaries}

We review here some basic properties of the first eigenvalue for the Dirichlet and the Neumann problems for $\Delta_{p}$.

Let $\Omega$ be a domain in $M$ and consider the Dirichlet problem:

$$
\begin{cases}\Delta_{p} f & =\mu|f|^{p-2} f \text { in } \Omega \\ f & =0\end{cases}
$$

Denote by $\mu_{1, p}(\Omega, g)$ the infimum of the set of eigenvalues for this problem. Here too, $\mu_{1, p}(\Omega, g)$ is a positive eigenvalue with the variational characterization

$$
\mu_{1, p}(\Omega, g)=\inf \left\{\frac{\int_{\Omega}|d f|^{p} \nu_{g}}{\int_{\Omega}|f|^{p} \nu_{g}} \mid f \in W_{0}^{1, p}(\Omega) \backslash\{0\}\right\} .
$$

The eigenfunctions associated to $\mu_{1, p}(\Omega, g)$ are essentially unique; they form a onedimensional space. Moreover, they have no zeros in $\Omega$ (see for instance [7]).

In [14, Veron proved the following result:

$$
\lambda_{1, p}(M, g)=\min _{\left(\omega_{1}, \omega_{2}\right)} \max \left\{\mu_{1, p}\left(\omega_{1}, g\right), \mu_{1, p}\left(\omega_{2}, g\right)\right\},
$$

where $\left(\omega_{1}, \omega_{2}\right)$ runs over the set of couples of nonempty disjoint open subsets of $M$.

Consider now the Neumann problem for $\Delta_{p}$ on $\Omega$ :

$$
\left\{\begin{array}{l}
\Delta_{p} f=\lambda|f|^{p-2} f \quad \text { in } \Omega \\
d f(\eta)=0 \quad \text { on } \partial \Omega
\end{array}\right.
$$

where $\eta$ denotes the exterior normal unit vector field to $\partial \Omega$.

Denote by $\lambda_{1, p}^{N}(\Omega, g)$ the infimum of the set of nonzero eigenvalues for this problem. By mimicking the proof of the closed case [14] we obtain that $\lambda_{1, p}^{N}(\Omega, g)$ is itself a positive eigenvalue with the variational characterization

$$
\lambda_{1, p}^{N}(\Omega, g)=\inf \left\{\left.\frac{\int_{\Omega}|d f|^{p} \nu_{g}}{\int_{\Omega}|f|^{p} \nu_{g}}\left|f \in W^{1, p}(\Omega) \backslash\{0\}, \int_{\Omega}\right| f\right|^{p-2} f \nu_{g}=0\right\} .
$$

A more general regularity result [12] says that the eigenfunctions for these problems are $C^{1}$, with gradient locally Hölder continuous. 


\section{RiEMANNIAN METRICS WITH ARBITRARILY LARGE $\lambda_{1, p}$}

Let $M$ be a compact connected manifold and denote by $\mathcal{M}(M)$ the space of Riemannian metrics of volume one on $M$.

It is known that we may diffeomorphically transform a local disc of $M$ into a "mushroom" attached to $M$ by a cylinder $C$. We can choose a Riemannian metric $g_{s} \in \mathcal{M}(M)$ such that some subset $C_{0} \subset C$ is the Riemannian product $[0, l] \times$ $S^{m-1}(s)\left(l, s\right.$ are positive constants and $S^{m-1}(s)$ denotes the $(m-1)$-dimensional Euclidean sphere of radius $s)$. Let $M_{1}$ and $M_{2}$ be the connected components of $M \backslash C_{0}$. We may choose $g_{s}$ such that $\operatorname{Vol}\left(M_{1}, g_{s}\right)=\operatorname{Vol}\left(M_{2}, g_{s}\right)$.

Let us consider a $C^{\infty}$ function $f$ equal to 1 on $M_{1}$, to -1 on $M_{2}$ and linear along $C_{0}$ (constant on the cross sections of $C_{0}$ ). Then $f$ satisfies the orthogonality condition $\int_{M}|f|^{p-2} f \nu_{g_{s}}=0$ and

$$
\lim _{s \rightarrow 0} \frac{\int_{M}|d f|^{p} \nu_{g_{s}}}{\int_{M}|f|^{p} \nu_{g_{s}}}=0 .
$$

The variational characterization for $\lambda_{1, p}\left(M, g_{s}\right) \operatorname{implies} \lim _{s \rightarrow 0} \lambda_{1, p}\left(M, g_{s}\right)=0$, i.e.:

Proposition 2.1. For any $p>1$,

$$
\inf _{g \in \mathcal{M}(M)} \lambda_{1, p}(M, g)=0 .
$$

Our main result is addressing the nonexistence of universal upper bounds for $\lambda_{1, p}$ on $\mathcal{M}(M)$ :

Theorem 2.2. For any $p>1$, every compact manifold $M$ of dimension $m \geq 3$ carries Riemannian metrics of volume one with $\lambda_{1, p}$ arbitrarily large.

In the case $p=2$ this Theorem is due to Colbois and Dodziuk [4].

The proof of Theorem 2.2 is divided into two parts: for $1<p<2$ the path we follow is reminiscent of the case $p=2$; however, new ideas are required since the arguments from the linear case do not apply when $p \neq 2$. In the case $p>2$, the proof follows directly from a monotonicity result.

Proof. I. The case $1<p \leq 2$

The main part of the proof consists in constructing a domain $D \subset S^{m}\left(S^{m}\right.$ denotes the $m$-dimensional unit Euclidean sphere) and a family of Riemannian metrics $\left(g_{n}\right)_{n \in \mathbb{N}}$ on $S^{m}$ such that

$$
\lim _{n \rightarrow \infty} \lambda_{1, p}^{N}\left(D, g_{n}\right)>0, \lim _{n \rightarrow \infty} \lambda_{1, p}\left(S^{m}, g_{n}\right)>0 \text { and } \lim _{n \rightarrow \infty} \operatorname{Vol}\left(D, g_{n}\right)=\infty
$$

We do this first on $S^{3}$ and then by following a path similar to [5] we show how to construct such domains and Riemannian metrics inductively on $S^{m}, m>3$ (can and $\widetilde{c a n}$ denote the canonical metrics on $S^{3}$, respectively $\left.C \mathbb{P}^{1}\right)$. For any $x \in S^{3}$, denote by $V_{x}=\operatorname{Ker}\left(d \pi_{x}\right)$ and by $H_{x}$ the space orthogonal to $V_{x}$ in $T_{x} S^{3}$ w.r.t. $\operatorname{can}_{x}$. Let $\left\{g_{t}\right\}_{t>0}$ be the Berger metrics defined by

$$
g_{t \mid V_{x} \times H_{x}}=0, \quad g_{t V_{x} \times V_{x}}=t^{2} \operatorname{can}_{\mid V_{x} \times V_{x}}, \quad g_{t \mid H_{x} \times H_{x}}=\operatorname{can}_{\mid H_{x} \times H_{x}} .
$$

The map $\pi$ is a Riemannian submersion from $\left(S^{3}, g_{t}\right)$ to $\left(C \mathbb{P}^{1}, \widetilde{c a n}\right)$ with totally geodesic fibers $F_{x}=\pi^{-1}(\pi(x))$ isometric to $\left(S^{1}, t^{2}\right.$ can $)$. 
Lemma 2.3. Let $A=\left\{\left(x_{1}, x_{2}, x_{3}, x_{4}\right) \in S^{3} \mid x_{3}^{2}+x_{4}^{2}<\frac{1}{4}\right\}$. Then for any $1<p \leq$ 2 ,

$$
\lambda_{1, p}^{N}\left(A, g_{t}\right) \geq \lambda_{1, p}\left(S^{3}, g_{t}\right) \geq 2^{-p} \mu_{1, p}\left(A, g_{t}\right) .
$$

Proof of Lemma 2.3. Fix $t$ and let $a=\left\{x \in S^{3} \mid x=\left(x_{1}, x_{2}, 0,0\right)\right\}, b=\{x \in$ $\left.S^{3} \mid x=\left(0,0, x_{3}, x_{4}\right)\right\}$ and $\mathcal{S}=S^{3} \backslash\{a \cup b\}$. Since $a$ and $b$ have codimension 2 in $S^{3}$, [1. Lemma 1] implies that $C_{0}^{\infty}(\mathcal{S})$ is dense in $W^{1,2}\left(S^{3}\right)$ and therefore in $W^{1, p}\left(S^{3}\right)$ for $1<p \leq 2$. We can then replace $W^{1, p}\left(S^{3}\right)$ by $C_{0}^{\infty}(\mathcal{S})$ in the variational characterization of $\lambda_{1, p}\left(S^{3}, g_{t}\right)$.

Parameterize $\mathcal{S}$ by

$$
\begin{aligned}
& \Phi:(0,1) \times[0,2 \pi) \times[0,2 \pi) \rightarrow \mathcal{S}, \\
& \Phi\left(r, \theta_{1}, \theta_{2}\right)=\left(\sqrt{1-r^{2}} \cos \theta_{1}, \sqrt{1-r^{2}} \sin \theta_{1}, r \cos \theta_{2}, r \sin \theta_{2}\right) .
\end{aligned}
$$

It is immediate that $\Phi\left(r, \theta_{1}, \theta_{2}\right) \in A \backslash a \Leftrightarrow r \in\left(0, \frac{1}{2}\right)$. Consider the diffeomorphism $\tau: A \backslash a \rightarrow \mathcal{S}$ defined by

$$
\tau\left(\Phi\left(r, \theta_{1}, \theta_{2}\right)\right)=\Phi\left(2 r, \theta_{1}, \theta_{2}\right) .
$$

Let $f \in C_{0}^{\infty}(\mathcal{S}) \backslash\{0\}$ with $\int_{S}|f|^{p-2} f \nu_{g_{t}}=0$. The function $f \circ \tau \in W_{0}^{1, p}(A \backslash a) \subset$ $W_{0}^{1, p}(A)$ and the variational characterization of $\mu_{1, p}\left(A, g_{t}\right)$ yields

$$
\mu_{1, p}\left(A, g_{t}\right) \leq \frac{\int_{A \backslash a}|d(f \circ \tau)|_{g_{t}}^{p} \nu_{g_{t}}}{\int_{A \backslash a}|f \circ \tau|^{p} \nu_{g_{t}}},
$$

where $|\cdot|_{g_{t}}$ denotes the Hilbert-Schmidt norm w.r.t. $g_{t}$. To evaluate the right side of inequality (2.4) note that for any $x \in \mathcal{S}, x=\Phi\left(r, \theta_{1}, \theta_{2}\right)$, the vectors

$$
\begin{aligned}
& v=d \Phi(0,1,1)=\left(-\sqrt{1-r^{2}} \sin \theta_{1}, \sqrt{1-r^{2}} \cos \theta_{1},-r \sin \theta_{2}, r \cos \theta_{2}\right), \\
& u_{1}=d \Phi\left(0,-\frac{r}{\sqrt{1-r^{2}}}, \frac{\sqrt{1-r^{2}}}{r}\right)=\left(r \sin \theta_{1},-r \cos \theta_{1},-\sqrt{1-r^{2}} \sin \theta_{2}, \sqrt{1-r^{2}} \cos \theta_{2}\right), \\
& u_{2}=d \Phi\left(\sqrt{1-r^{2}}, 0,0\right)=\left(-r \cos \theta_{1},-r \sin \theta_{1}, \sqrt{1-r^{2}} \cos \theta_{2}, \sqrt{1-r^{2}} \sin \theta_{2}\right)
\end{aligned}
$$

form an orthonormal basis of $T_{x} S^{3}$ w.r.t. $c a n_{x}$. Moreover $v=\left.\frac{d \mathbf{c}}{d s}\right|_{s=0}$ where $\mathbf{c}:[0,2 \pi) \rightarrow F_{x}, \mathbf{c}(s)=\Phi\left(r, \theta_{1}+s, \theta_{2}+s\right)$. It is easy to see that $\pi \circ \mathbf{c}(s)=\pi(x)$ for all $s$ and therefore $v \in \operatorname{Ker}\left(d \pi_{x}\right)=V_{x}$. Hence, $\left\{t^{-1} v, u_{1}, u_{2}\right\}$ is an orthonormal basis of $T_{x} S^{3}$ w.r.t. $g_{t}$, for any $t>0$. On the other hand, (2.3) implies

$$
d \tau(v)=v, \quad d \tau\left(u_{1}\right)=u_{1}, \quad d \tau\left(u_{2}\right)=2 u_{2} .
$$

We have

$$
\begin{aligned}
|d(f \circ \tau)|_{g_{t}}^{p} & =\left(\left|d(f \circ \tau)\left(t^{-1} v\right)\right|^{2}+\left|d(f \circ \tau)\left(u_{1}\right)\right|^{2}+\left|d(f \circ \tau)\left(u_{2}\right)\right|^{2}\right)^{\frac{p}{2}} \\
& =\left(\left|d f\left(t^{-1} v\right)\right|^{2}+\left|d f\left(u_{1}\right)\right|^{2}+\left|d f\left(2 u_{2}\right)\right|^{2}\right)^{\frac{p}{2}} \circ \tau \\
& \leq 2^{p}|d f|_{g_{t}}^{p} \circ \tau .
\end{aligned}
$$


Integrating over $A \backslash a$ we obtain

$$
\begin{aligned}
\int_{A \backslash a}|d(f \circ \tau)|_{g_{t}}^{p} \nu_{g_{t}} & \leq 2^{p} \int_{A \backslash a}|d f|_{g_{t}}^{p} \circ \tau \nu_{g_{t}} \\
& =2^{p-1} \int_{A \backslash a}|d f|_{g_{t}}^{p} \circ \tau \nu_{\tau^{*} g_{t}} \\
& =2^{p-1} \int_{\mathcal{S}}|d f|_{g_{t}}^{p} \nu_{g_{t}}
\end{aligned}
$$

(where in the first equality we have used $\nu_{\tau^{*} g_{t}}=2 \nu_{g_{t}}$, which follows immediately from (2.5)). Similarly

$$
\int_{A \backslash a}|f \circ \tau|^{p} \nu_{g_{t}}=\frac{1}{2} \int_{\mathcal{S}}|f|^{p} \nu_{g_{t}} .
$$

Equations (2.4), (2.6) and (2.7) imply that

$$
\mu_{1, p}\left(A, g_{t}\right) \leq 2^{p} \frac{\int_{\mathcal{S}}|d f|^{p} \nu_{g_{t}}}{\int_{\mathcal{S}}|f|^{p} \nu_{g_{t}}}
$$

Taking now the infimum over all $f \in C_{0}^{\infty}(\mathcal{S}) \backslash\{0\}$ with $\int_{\mathcal{S}}|f|^{p-2} f \nu_{g_{t}}=0$ we obtain

$$
\mu_{1, p}\left(A, g_{t}\right) \leq 2^{p} \lambda_{1, p}\left(S^{3}, g_{t}\right) .
$$

To prove the left inequality in Lemma 2.3 note that since $C^{\infty}(\bar{A})$ is dense in $W^{1, p}(A)$, by the same argument as above we obtain that $C_{0}^{\infty}(\bar{A} \backslash a)$ is dense in $W^{1, p}(A)$. We can thus replace $W^{1, p}(A)$ by $C_{0}^{\infty}(\bar{A} \backslash a)$ in the variational characterization for $\lambda_{1, p}^{N}\left(A, g_{t}\right)$. Let $f \in C_{0}^{\infty}(\bar{A} \backslash a) \backslash\{0\}$ with $\int_{A \backslash a}|f|^{p-2} f \nu_{g_{t}}=0$. Let $B=\left\{\left(x_{1}, x_{2}, x_{3}, x_{4}\right) \in S^{3} \mid x_{3}^{2}+x_{4}^{2}>\frac{1}{4}\right\}$ and consider the diffeomorphism $\Psi: \mathcal{S} \rightarrow \mathcal{S}$ given by

$$
\Psi\left(\Phi\left(r, \theta_{1}, \theta_{2}\right)\right)=\Phi\left(1-r, \theta_{1}, \theta_{2}\right) .
$$

Extend $f$ into a function $F \in W_{0}^{1, p}(\mathcal{S})$ defined by

$$
F(x)= \begin{cases}f(x) & \text { if } x \in \bar{A} \backslash a, \\ f \circ \Psi(x) & \text { if } x \in B \backslash b .\end{cases}
$$

Equation (2.8) implies $d \Psi(v)=v, d \Psi\left(u_{1}\right)=u_{1}$ and $d \Psi\left(u_{2}\right)=-u_{2}$. Hence, $\Psi$ is an isometry of $(\mathcal{S}$, can $)$; in fact, since $v$ is invariant by $d \Psi, \Psi$ is an isometry of $\left(\mathcal{S}, g_{t}\right)$, for any $t>0$. In particular, given that $\Psi(A \backslash a)=B \backslash b$, we have

$$
\begin{gathered}
\int_{\mathcal{S}}|d F|_{g_{t}}^{p} \nu_{g_{t}}=2 \int_{A \backslash a}|d f|_{g_{t}}^{p} \nu_{g_{t}}, \quad \int_{\mathcal{S}}|F|^{p} \nu_{g_{t}}=2 \int_{A \backslash a}|f|^{p} \nu_{g_{t}}, \\
\int_{\mathcal{S}}|F|^{p-2} F \nu_{g_{t}}=2 \int_{A \backslash a}|f|^{p-2} f \nu_{g_{t}}=0 .
\end{gathered}
$$

The variational characterization for $\lambda_{1, p}\left(S^{3}, g_{t}\right)$ yields

$$
\lambda_{1, p}\left(S^{3}, g_{t}\right) \leq \frac{\int_{\mathcal{S}}|d F|_{g_{t}}^{p} \nu_{g_{t}}}{\int_{\mathcal{S}}|F|^{p} \nu_{g_{t}}}=\frac{\int_{A \backslash a}|d f|_{g_{t}}^{p} \nu_{g_{t}}}{\int_{A \backslash a}|f|^{p} \nu_{g_{t}}} .
$$

Taking now the infimum over all $f \in C_{0}^{\infty}(\bar{A} \backslash a) \backslash\{0\}$ with $\int_{A}|f|^{p-2} f \nu_{g_{t}}=0$ we obtain $\lambda_{1, p}\left(S^{3}, g_{t}\right) \leq \lambda_{1, p}^{N}\left(A, g_{t}\right)$. This ends the proof of Lemma 2.3 
Lemma 2.4. Let $A$ be as in Lemma [2.3. Then for any $1<p \leq 2$,

$$
\mu_{1, p}\left(A, g_{t}\right) \geq 2^{\frac{p}{2}-1} \lambda_{1, p}\left(C \mathbb{P}^{1}, \widetilde{c a n}\right) .
$$

Proof of Lemma [2.4. Fix $t$ and let $f \in W_{0}^{1, p}(A)$ be an eigenfunction for $\mu_{1, p}\left(A, g_{t}\right)$. Then $f$ has no zeros in $A$, and we may assume that $f>0$ in $A$. It is easy to see that for any $x \in A, F_{x} \subset A$. We can construct therefore a positive function $\tilde{f}: \pi(A) \subset C \mathbb{P}^{1} \rightarrow \mathbb{R}$ by setting

$$
\tilde{f}(\pi(x))^{p}=\int_{F_{x}} f^{p} \nu_{g_{t}} .
$$

Let $u_{1}, u_{2}$ be two vector fields such that for all $x \in A,\left\{u_{1}, u_{2}\right\}$ is an orthonormal basis of $H_{x}$ w.r.t. $g_{t}$. Then $\left\{\tilde{u}_{1}=d \pi\left(u_{1}\right), \tilde{u}_{2}=d \pi\left(u_{2}\right)\right\}$ is an orthonormal basis of $T_{\pi(x)} C \mathbb{P}^{1}$ w.r.t. $\widetilde{c a n}$. Given that the fibers $F_{x}$ are totally geodesic, we can apply Lemma 3.14 in [2] to the function $f^{p}$ and the vector field $u_{1}$ and obtain

$$
p \tilde{f}^{p-1} d \tilde{f}\left(\tilde{u}_{1}\right)=p \int_{F_{x}} f^{p-1} d f\left(u_{1}\right) \nu_{g_{t}} .
$$

The Hölder inequality yields

$$
\begin{aligned}
\tilde{f}^{p-1}\left|d \tilde{f}\left(\tilde{u}_{1}\right)\right| & \leq \int_{F_{x}} f^{p-1}\left|d f\left(u_{1}\right)\right| \nu_{g_{t}} \\
& \leq\left(\int_{F_{x}} f^{p} \nu_{g_{t}}\right)^{\frac{p-1}{p}}\left(\int_{F_{x}}\left|d f\left(u_{1}\right)\right|^{p} \nu_{g_{t}}\right)^{\frac{1}{p}} \\
& =\tilde{f}^{p-1}\left(\int_{F_{x}}\left|d f\left(u_{1}\right)\right|^{p} \nu_{g_{t}}\right)^{\frac{1}{p}} .
\end{aligned}
$$

Since $\tilde{f}>0$, it follows that $\left|d \tilde{f}\left(\tilde{u}_{1}\right)\right|^{p} \leq \int_{F_{x}}\left|d f\left(u_{1}\right)\right|^{p} \nu_{g_{t}}$. In the same way we obtain $\left|d \tilde{f}\left(\tilde{u}_{2}\right)\right|^{p} \leq \int_{F_{x}}\left|d f\left(u_{2}\right)\right|^{p} \nu_{g_{t}}$. Hence

$$
\begin{aligned}
|d \tilde{f}|_{\text {can }}^{p} & =\left(\left|d \tilde{f}\left(\tilde{u}_{1}\right)\right|^{2}+\left|d \tilde{f}\left(\tilde{u}_{2}\right)\right|^{2}\right)^{\frac{p}{2}} \leq\left|d \tilde{f}\left(\tilde{u}_{1}\right)\right|^{p}+\left|d \tilde{f}\left(\tilde{u}_{2}\right)\right|^{p} \\
& \leq \int_{F_{x}}\left|d f\left(u_{1}\right)\right|^{p}+\left|d f\left(u_{2}\right)\right|^{p} \nu_{c a n} \\
& \leq 2^{1-\frac{p}{2}} \int_{F_{x}}\left(\left|d f\left(u_{1}\right)\right|^{2}+\left|d f\left(u_{2}\right)\right|^{2}\right)^{\frac{p}{2}} \nu_{c a n} \\
& \leq 2^{1-\frac{p}{2}} \int_{F_{x}}|d f|_{g_{t}}^{p} \nu_{\text {can }}
\end{aligned}
$$

(where we have used the inequality: $(a+b)^{\frac{p}{2}} \leq a^{\frac{p}{2}}+b^{\frac{p}{2}} \leq 2^{1-\frac{p}{2}}(a+b)^{\frac{p}{2}}$ for any $a, b>0$ and $1<p \leq 2)$. Integrating (2.9) and (2.10) over $\pi(A)$ we obtain

$$
\int_{\pi(A)}|\tilde{f}|^{p} \nu_{\widetilde{c a n}}=\int_{A}|f|^{p} \nu_{g_{t}} \text { and } \int_{\pi(A)}|d \tilde{f}|^{p} \nu_{\widetilde{c a n}} \leq 2^{1-\frac{p}{2}} \int_{A}|d f|_{g_{t}}^{p} \nu_{g_{t}} .
$$

In particular, (2.11) implies that $\tilde{f} \in W^{1, p}(\pi(A))$. In fact, since $f \in W_{0}^{1, p}(A)$ and $F_{x} \subset \partial A$ for any $x \in \partial A$, it is immediate from (2.9) that $\tilde{f} \in W_{0}^{1, p}(\pi(A))$. The variational characterization for $\mu_{1, p}(\pi(A), \widetilde{c a n})$ and (2.11) imply then

$$
\mu_{1, p}(\pi(A), \widetilde{c a n}) \leq \frac{\int_{\pi(A)}|d \tilde{f}|^{p} \nu_{\widetilde{c a n}}}{\int_{\pi(A)}|\tilde{f}|^{p} \nu_{\widetilde{c a n}}} \leq 2^{1-\frac{p}{2}} \frac{\int_{A}|d f|_{g_{t}}^{p} \nu_{g_{t}}}{\int_{A}|f|^{p} \nu_{g_{t}}}=2^{1-\frac{p}{2}} \mu_{1, p}\left(A, g_{t}\right) \text {. }
$$


In the same way we can prove that $\mu_{1, p}(\pi(B), \widetilde{c a n}) \leq 2^{1-\frac{p}{2}} \mu_{1, p}\left(B, g_{t}\right)$, where $B$ is as in Lemma 2.3. The map $\Psi$ is an isometry between $A \backslash a$ and $B \backslash b$ and therefore it is straightforward that $\mu_{1, p}\left(A, g_{t}\right)=\mu_{1, p}\left(B, g_{t}\right)$. Since the open sets $\pi(A)$ and $\pi(B)$ are disjoint, (1.1) and (2.12) imply

$$
\lambda_{1, p}\left(C \mathbb{P}^{1}, \widetilde{c a n}\right) \leq \max \left\{\mu_{1, p}(\pi(A), \widetilde{c a n}), \mu_{1, p}(\pi(B), \widetilde{\operatorname{can}}\} \leq 2^{1-\frac{p}{2}} \mu_{1, p}\left(A, g_{t}\right) .\right.
$$

Combining Lemma 2.3 and Lemma 2.4 we obtain

$$
\lambda_{1, p}^{N}\left(A, g_{t}\right) \geq \lambda_{1, p}\left(S^{3}, g_{t}\right) \geq 2^{-\frac{p}{2}-1} \lambda_{1, p}\left(C \mathbb{P}^{1}, \widetilde{c a n}\right) .
$$

Finally, since $\nu_{g_{t}}=t \nu_{g}$ we have $\operatorname{Vol}\left(A, g_{t}\right) \rightarrow \infty$ as $t \rightarrow \infty$. In particular, we can find a sequence $t_{n} \rightarrow \infty$ such that the metrics $g_{n}:=g_{t_{n}}$ and the domain $A$ satisfy (2.1) on $S^{3}$.

We will now construct a domain $D \subset S^{m+1}$ and a family of metrics on $S^{m+1}$ with the property (2.1), given such domain and metrics on $S^{m}$. To do this we follow the general idea from [5].

Let $D \subset S^{m}$ and $\left(g_{n}\right)_{n \in \mathbb{N}}$ with property (2.1). (In fact we need only the last two assumptions in (2.1) for the construction on $S^{m+1}$.) Let $C=S^{m} \times(0,1)$ endowed with the metric $h_{n}=g_{n} \times d r^{2}$ where $d r^{2}$ denotes the Euclidean metric on $(0,1)$. Obviously $\operatorname{Vol}\left(C, h_{n}\right)=\operatorname{Vol}\left(S^{m}, g_{n}\right)>\operatorname{Vol}\left(D, g_{n}\right)$. Let $f_{n}$ be an eigenfunction for $\lambda_{1, p}^{N}\left(C, h_{n}\right)$ and denote by $f_{n}^{+}, f_{n}^{-}$its positive, respectively, its negative part. In the same way as in [8 Lemma 3], it is straightforward that

$$
\lambda_{1, p}^{N}\left(C, h_{n}\right)=\frac{\int_{C}\left|d f_{n}\right|_{h_{n}}^{p} \nu_{h_{n}}}{\int_{C}\left|f_{n}\right|^{p} \nu_{h_{n}}}=\frac{\int_{C}\left|d f_{n}^{+}\right|_{h_{n}}^{p} \nu_{h_{n}}}{\int_{C}\left|f_{n}^{+}\right|^{p} \nu_{h_{n}}}=\frac{\int_{C}\left|d f_{n}^{-}\right|_{h_{n}}^{p} \nu_{h_{n}}}{\int_{C}\left|f_{n}^{-}\right|^{p} \nu_{h_{n}}}
$$

After multiplying $f_{n}^{+}$and $f_{n}^{-}$by constants we may assume that $\int_{C}\left|f_{n}^{+}\right|^{p} \nu_{h_{n}}=$ $\int_{C}\left|f_{n}^{-}\right|^{p} \nu_{h_{n}}=1$. Then

$$
\lambda_{1, p}^{N}\left(C, h_{n}\right)=\int_{C}\left|d f_{n}^{+}\right|_{h_{n}}^{p} \nu_{h_{n}}=\int_{C}\left|d f_{n}^{-}\right|_{h_{n}}^{p} \nu_{h_{n}} .
$$

Consider the positive functions $\tilde{f}_{n}^{+}(r)$ and $\tilde{f}_{n}^{-}(r)$ defined by

$$
\tilde{f}_{n}^{+}(r)^{p}=\int_{S^{m}}\left|f_{n}^{+}\right|^{p}(\cdot, r) \nu_{g_{n}} \quad \text { and } \quad \tilde{f}_{n}^{-}(r)^{p}=\int_{S^{m}}\left|f_{n}^{-}\right|^{p}(\cdot, r) \nu_{g_{n}} .
$$

As in the proof of Lemma 2.4, we can apply Lemma 3.14 of [2] to the function $\left(\tilde{f}_{n}^{+}\right)^{p}$ and the vector field $\frac{\partial}{\partial r}$ and obtain

$$
\left|\left(\tilde{f}_{n}^{+}\right)^{\prime}\right|^{p} \leq \int_{S^{m}}\left|d f_{n}^{+}\left(\frac{\partial}{\partial r}\right)\right|{ }^{p} \nu_{g_{n}} .
$$

Integrating (2.14) and (2.15) we get $\int_{0}^{1}\left|\tilde{f}_{n}^{+}\right|^{p} d r=\int_{C}\left|f_{n}^{+}\right|^{p} \nu_{h_{n}}=1$ and

$$
\int_{0}^{1}\left|\left(\tilde{f}_{n}^{+}\right)^{\prime}\right|^{p} d r \leq \int_{C}\left|d f_{n}^{+}\left(\frac{\partial}{\partial r}\right)\right|{ }^{p} \nu_{h_{n}} \leq \int_{C}\left|d f_{n}^{+}\right|_{h_{n}}^{p} \nu_{h_{n}}=\lambda_{1, p}^{N}\left(C, h_{n}\right) .
$$

Suppose now that $\lim _{n \rightarrow \infty} \lambda_{1, p}^{N}\left(C, h_{n}\right)=0$. Then (2.16) implies that the sequence $\left(\tilde{f}_{n}^{+}\right)_{n \in \mathbb{N}}$ is bounded in $W^{1, p}(0,1)$. After extracting a subsequence, we may assume 
that there exists a function $\tilde{f}_{0}^{+} \in W^{1, p}(0,1)$ such that $\tilde{f}_{n}^{+}$converges to $\tilde{f}_{0}^{+}$weakly in $W^{1, p}(0,1)$ and strongly in $L^{p}(0,1)$. The weak convergence and (2.16) yield $\int_{0}^{1}\left|\left(\tilde{f}_{0}^{+}\right)^{\prime}\right|^{p} d r \leq \liminf _{n \rightarrow \infty} \int_{0}^{1}\left|\left(\tilde{f}_{n}^{+}\right)^{\prime}\right|^{p} d r \leq \lim _{n \rightarrow \infty} \lambda_{1, p}^{N}\left(C, h_{n}\right)=0 \Rightarrow \tilde{f}_{0}^{+}$is constant .

The strong convergence implies that $\int_{0}^{1}\left|\tilde{f}_{0}^{+}\right|^{p} d r=\lim _{n \rightarrow \infty} \int_{0}^{1}\left|\tilde{f}_{n}^{+}\right|^{p} d r=1$. Since $\tilde{f}_{0}^{+}$is constant, we have $\tilde{f}_{0}^{+} \equiv 1$, i.e. $\tilde{f}_{n}^{+} \rightarrow 1$ in $L^{p}(0,1)$. Similarly, after extracting a subsequence again, we obtain $\tilde{f}_{n}^{-} \rightarrow 1$ in $L^{p}(0,1)$. In particular,

$$
\lim _{n \rightarrow \infty} \int_{S^{m}}\left|f_{n}^{+}\right|^{p}(\cdot, r) \nu_{g_{n}}=\lim _{n \rightarrow \infty} \int_{S^{m}}\left|f_{n}^{-}\right|^{p}(\cdot, r) \nu_{g_{n}}=1 \text { for almost all } r .
$$

On the other hand, (2.13) implies

$$
\lambda_{1, p}^{N}\left(C, h_{n}\right)=\int_{0}^{1} \int_{S^{m}}\left|d f_{n}^{+}\right|_{h_{n}}^{p} \nu_{g_{n}} d r \geq \int_{0}^{1} \int_{S^{m}}\left|d f_{n}^{+}\right|_{g_{n}}^{p} \nu_{g_{n}} d r
$$

and a similar inequality for $f_{n}^{-}$. Hence

$$
\lim _{n \rightarrow \infty} \int_{S^{m}}\left|d f_{n}^{+}\right|_{g_{n}}^{p}(\cdot, r) \nu_{g_{n}}=\int_{S^{m}}\left|d f_{n}^{-}\right|_{g_{n}}^{p}(\cdot, r) \nu_{g_{n}}=0 \text { for almost all } r .
$$

Combining (2.17) and (2.18) we obtain

$$
\lim _{n \rightarrow \infty} \frac{\int_{S^{m}}\left|d f^{+}\right|_{g_{n}}^{p}(\cdot, r) \nu_{g_{n}}}{\int_{S^{m}}\left|f^{+}\right|^{p}(\cdot, r) \nu_{g_{n}}}=\lim _{n \rightarrow \infty} \frac{\int_{S^{m}}\left|d f^{-}\right|_{g_{n}}^{p}(\cdot, r) \nu_{g_{n}}}{\int_{S^{m}}\left|f^{-}\right|^{p}(\cdot, r) \nu_{g_{n}}}=0 \text { for almost all } r .
$$

Fix $r$ and denote by $\omega_{n}^{+}, \omega_{n}^{-}$the (disjoint) supports in $S^{m}$ of $f_{n}^{+}(\cdot, r)$ and $f_{n}^{-}(\cdot, r)$ respectively. The variational characterization for $\mu_{1, p}\left(\omega_{r}^{+}, g_{n}\right)$ and $\mu_{1, p}\left(\omega_{r}^{-}, g_{n}\right)$ together with (1.1) implies

$$
\begin{aligned}
& \max \left\{\frac{\int_{S^{m}}\left|d f_{n}^{+}\right|_{g_{n}}^{p}(\cdot, r) \nu_{g_{n}}}{\int_{S^{m}}\left|f_{n}^{+}\right|^{p}(\cdot, r) \nu_{g_{n}}}, \frac{\int_{S^{m}}\left|d f_{n}^{-}\right|_{g_{n}}^{p}(\cdot, r) \nu_{g_{n}}}{\int_{S^{m}}\left|f^{-}\right|^{p}(\cdot, r) \nu_{g_{n}}}\right\} \\
& \geq \max \left\{\mu_{1, p}\left(\omega_{n}^{+}, g_{n}\right), \mu_{1, p}\left(\omega_{n}^{-}, g_{n}\right)\right\} \\
& \geq \lambda_{1, p}\left(S^{m}, g_{n}\right) .
\end{aligned}
$$

Equation (2.19) implies then that $\lim _{n \rightarrow \infty} \lambda_{1, p}\left(S^{m}, g_{n}\right)=0$. This contradicts our choice of $g_{n}$. It follows that $\lim _{n \rightarrow \infty} \lambda_{1, p}^{N}\left(C, h_{n}\right) \neq 0$ and therefore (after extracting a subsequence) we may assume $\lim _{n \rightarrow \infty} \lambda_{1, p}^{N}\left(C, h_{n}\right)>0$.

To conclude our construction on $S^{m+1}$ we need the first two assertions of the following technical result [9]:

Lemma 2.5. Let $(M, g)$ be a compact Riemannian manifold, $p>1$ and $\Omega \subset M a$ domain with Lipschitzian boundary. Then for any $\delta>0$, there exists a metric $\tilde{g}$ on $M$ such that:

- $g_{\mid \Omega}=\tilde{g}_{\mid \Omega}$,

- $\lambda_{1, p}(M, \tilde{g})>\lambda_{1, p}^{N}(\Omega, g)-\delta$,

- $|\operatorname{Vol}(M, \tilde{g})-\operatorname{Vol}(\Omega, g)|<\delta$.

We can now glue two caps to $C$ to obtain a manifold diffeomorphic to $S^{m+1}$. Let $2 \delta=\lim _{n \rightarrow \infty} \lambda_{1, p}^{N}\left(C, h_{n}\right)>0$. Lemma 2.5 implies that we can extend $h_{n}$ into a metric $\tilde{h}_{n}$ on the whole of $S^{m+1}$ such that $\lambda_{1, p}\left(S^{m+1}, \tilde{h}_{n}\right)>\lambda_{1, p}^{N}\left(C, h_{n}\right)-\delta$. Then 
$C$ and (after extracting a subsequence) the family $\left(\tilde{h}_{n}\right)_{n \in \mathbb{N}}$ will satisfy the property (2.1) on $S^{m+1}$.

Now let $M$ be an arbitrarily compact manifold of dimension $m \geq 3$. Consider a domain $D \subset S^{m}$ and a family of Riemannian metrics $\left(g_{n}\right)_{n \in \mathbb{N}}$ on $S^{m}$ with the property (2.1). Let $x_{0} \in S^{m}$ such that $x_{0} \notin D$ and let $U$ be a small open set in $M$ diffeomorphic to $S^{m} \backslash\left\{x_{0}\right\}$ (since $S^{m} \backslash\left\{x_{0}\right\}$ is diffeomorphic to a ball in $\mathbb{R}^{m}$, it suffices to take $U$ diffeomorphic to a ball). We can pull back the metric $g_{n}$ via this diffeomorphism into a metric $\bar{g}_{n}$ on $U$. Then $\left(D, g_{n}\right)$ is isometric to $\left(\Omega, \bar{g}_{n}\right)$, where $\Omega$ is some subset of $U$. We have

$$
\lambda_{1, p}^{N}\left(D, g_{n}\right)=\lambda_{1, p}^{N}\left(\Omega, \bar{g}_{n}\right) \text { and } \operatorname{Vol}\left(\Omega, \bar{g}_{n}\right)=\operatorname{Vol}\left(D, g_{n}\right) \rightarrow \infty \text { as } n \rightarrow \infty .
$$

Now let $2 \delta=\lim _{n \rightarrow \infty} \lambda_{1, p}^{N}\left(\Omega, \bar{g}_{n}\right)>0$. We can perturb each $\bar{g}_{n}$ outside $\Omega$ as in Lemma 2.5 and obtain a family of metrics $\left(\tilde{g}_{n}\right)_{n \in \mathbb{N}}$ on $M$ such that $\lambda_{1, p}\left(M, \tilde{g}_{n}\right)>$ $\lambda_{1, p}^{N}\left(\Omega, \bar{g}_{n}\right)-\delta$. Since $\operatorname{Vol}\left(M, \tilde{g}_{n}\right)>\operatorname{Vol}\left(\Omega, \bar{g}_{n}\right)$ we have $\lim _{n \rightarrow \infty} \operatorname{Vol}\left(M, \tilde{g}_{n}\right)=\infty$. Now let $\widehat{g}_{n}=\operatorname{Vol}\left(M, \tilde{g}_{n}\right)^{-\frac{2}{m}} \tilde{g}_{n}$. Then $\widehat{g}_{n} \in \mathcal{M}(M)$ and

$$
\lambda_{1, p}\left(M, \widehat{g}_{n}\right)=\operatorname{Vol}\left(M, \tilde{g}_{n}\right)^{\frac{p}{m}} \lambda_{1, p}\left(M, \tilde{g}_{n}\right) \rightarrow \infty \text { as } n \rightarrow \infty .
$$

(If $M=S^{m}$, it suffices to construct the metrics $\widehat{g}_{n}=\operatorname{Vol}\left(S^{m}, g_{n}\right)^{-\frac{2}{m}} g_{n} \in \mathcal{M}\left(S^{m}\right)$ to obtain $\lim _{n \rightarrow \infty} \lambda_{1, p}\left(S^{m}, \widehat{g}_{n}\right)=\infty$.) This ends the proof of the case $1<p \leq 2$.

II. The case $p>2$

In this case the proof follows directly from the following monotonicity result:

Proposition 2.6. Let $(M, g)$ be a compact Riemannian manifold. Then the map

$$
p \mapsto p \lambda_{1, p}(M, g)^{\frac{1}{p}}
$$

is strictly increasing on $(1, \infty)$.

Proof of Proposition 2.6. Let $1<p<q$ and $A_{1}, A_{2}$ be the nodal domains of an eigenfunction for $\lambda_{1, q}(M, g)$. Then $\lambda_{1, q}(M, g)=\mu_{1, q}\left(A_{1}, g\right)=\mu_{1, q}\left(A_{2}, g\right)[8]$.

For any bounded domain $\Omega$ of $M$, Lindqvist [7] proved that the map $p \mapsto$ $p \mu_{1, p}(\Omega)^{\frac{1}{p}}$ is strictly increasing on $] 1, \infty[$. We then have

$$
p \mu_{1, p}\left(A_{1}, g\right)^{\frac{1}{p}}<q \mu_{1, q}\left(A_{1}, g\right)^{\frac{1}{q}} \text { and } p \mu_{1, p}\left(A_{2}, g\right)^{\frac{1}{p}}<q \mu_{1, q}\left(A_{2}, g\right)^{\frac{1}{q}} .
$$

On the other hand, (1.1) implies $\lambda_{1, p}(M, g) \leq \max \left\{\mu_{1, p}\left(A_{1}, g\right), \mu_{1, p}\left(A_{2}, g\right)\right\}$. Hence

$$
\begin{aligned}
p \lambda_{1, p}(M, g)^{\frac{1}{p}} & \leq \max \left\{p \mu_{1, p}\left(A_{1}, g\right)^{\frac{1}{p}}, p \mu_{1, p}\left(A_{2}, g\right)^{\frac{1}{p}}\right\} \\
& <\max \left\{q \mu_{1, q}\left(A_{1}, g\right)^{\frac{1}{q}}, q \mu_{1, q}\left(A_{2}, g\right)^{\frac{1}{q}}\right\} \\
& =q \mu_{1, q}\left(A_{1}, g\right)^{\frac{1}{q}} \\
& =q \lambda_{1, q}(M, g)^{\frac{1}{q}} .
\end{aligned}
$$

Finally, Proposition 2.6 implies that for any $p>2$,

$$
\lambda_{1, p}(M, g)>\left(\frac{2}{p}\right)^{p}\left(\lambda_{1,2}(M, g)\right)^{\frac{p}{2}} .
$$

Since the functional $g \rightarrow \lambda_{1,2}(M, g)$ is not bounded on $\mathcal{M}(M)$ it follows that for any $p>2$ the functional $g \rightarrow \lambda_{1, p}(M, g)$ is also not bounded on $\mathcal{M}(M)$. This ends the proof of Theorem 2.2. 
Remark 2.7. Proposition 2.6 provides a simple method for obtaining lower bounds for $\lambda_{1, p}(M, g), p>2$, in terms of other geometric invariants. For example, it is known that $\lambda_{1,2}(M, g)>\frac{h_{(M, g)}^{2}}{4}$ where $h_{(M, g)}$ is the Cheeger isoperimetric constant of $(M, g)$. We then have

$$
\lambda_{1, p}(M, g)>\left(\frac{2}{p}\right)^{p}\left(\lambda_{1,2}(M, g)\right)^{\frac{p}{2}}>\left(\frac{h_{(M, g)}}{p}\right)^{p} \text { for any } p>2 .
$$

Note that in $[8]$ we proved that $\lambda_{1, p}(M, g)>\left(\frac{h_{(M, g)}}{p}\right)^{p}$ for any $p>1$.

\section{REFERENCES}

1. C. Anné, Spectre du Laplacien et écrasement d'anses, Ann. Scient. Éc. Norm. Sup., 4 série, 20 (1987), 271-280. MR0911759 (89m:58200)

2. Bérard Bergery, L. \& Bourguignon J-P., Laplacians and riemannian submersions with totally geodesic fibres, Ilinois Journal of Mathematics, 26 (1982), 181-200. MR 0650387 (84m:58153)

3. J. Cheeger, A lower bound for the smallest eigenvalue of the Laplacian, Problems in Analysis, A Symposium in Honor of S. Bochner, Princeton University Press, 1970, pp. 195-199. MR0402831 (53:6645)

4. B. Colbois \& J. Dodziuk, Riemannian metrics with large $\lambda_{1}$, Proceedings of the American Mathematical Society, Vol 122, n. 3 (1994), 905-906. MR1213857(95a:58130)

5. J. Dodziuk, Nonexistence of universal upper bounds for the first positive eigenvalue of the Laplace-Beltrami operator, Geometry of the Spectrum (Seattle, Wa, 1993) 109-114, Contemp. Math., 173, Amer. Math. Soc. Providence, RI, 1994. MF1298199 (95m:53052)

6. J. P. Garzia Azorero, \& I. Peral Alonso, Existence and nonuniqueness for the p-Laplacian eigenvalues, Comm. Part. Diff. Equ., 12 (1987), 1389-1430. MR0912211 (89e:35058)

7. P. Lindqvist, On the equation $\operatorname{div}\left(|\nabla u|^{p-2} \nabla u\right)+\lambda|u|^{p-2} u=0$, Proc. Am. Math. Soc., 109, No 1 (1990), 157-164. MR1007505 (90h:35088)

8. A-M. Matei, First eigenvalue for the p-Laplace operator, Nonlinear Anal., 39 (2000), 10511068. MR1735181 (2001a:58041)

9. A-M. Matei, The effect of perturbations on the first eigenvalue of the p-Laplacian, C. R. Acad. Sci. Paris, Ser. I 335 (2002) 255-258. MR1933668 (2003h:58044)

10. H. Muto, The first eigenvalue of the Laplacian on even dimensional spheres, Tohoku Math. J., 32 (1980), 427-432. MF0590038 (82a:58052)

11. S. Tanno, The first eigenvalue of the laplacian on spheres, Tôhoku Math. J., 31 (1979), 179-185. MR0538918 (80g:58050)

12. P. Tolksdorf, Regularity for a more general class of quasilinear elliptic equations, J. Diff. Equ., 51 (1984), 126-150. MR 0727034 (85g:35047)

13. H. Urakawa, On the least positive eigenvalue of the Laplacian for compact group manifolds, J. Math. Soc. Japan, 31 (1979), 209-226. MR0519046 (80e:58046)

14. L. Veron, Some Existence and Uniqueness Results for Solution of Some Quasilinear Elliptic Equations on Compact Riemannian Manifolds, Colloquia Mathematica Societatis János Bolyai, 62, P.D.E., Budapest (1991), 317-352. MR1468764

15. Y. Xu, Diverging eigenvalues and collapsing Riemannian metrics, preprint, Institute for Advanced Studies, October 1992.

Department of Mathematics and Computer Science, Loyola University New Orleans, 6363 St. Charles Avenue, New Orleans, Louisiana 70118

E-mail address: amatei@loyno.edu 